\title{
Double photoionization of atomic helium and its isoelectronic partners at $x$-ray energies
}

\section{Citation}

Dalgarno, A., and H. R. Sadeghpour. 1992. “Double Photoionization of Atomic Helium and Its Isoelectronic Partners at X-Ray Energies." Physical Review A 46 (7): R3591-93. https:// doi.org/10.1103/physreva.46.r3591.

\section{Permanent link}

http://nrs.harvard.edu/urn-3:HUL.InstRepos:41417400

\section{Terms of Use}

This article was downloaded from Harvard University's DASH repository, and is made available under the terms and conditions applicable to Other Posted Material, as set forth at http:// nrs.harvard.edu/urn-3:HUL.InstRepos:dash.current.terms-of-use\#LAA

\section{Share Your Story}

The Harvard community has made this article openly available.

Please share how this access benefits you. Submit a story.

Accessibility 


\title{
Double photoionization of atomic helium and its isoelectronic partners at x-ray energies
}

\author{
A. Dalgarno and H. R. Sadeghpour \\ Harvard-Smithsonian Center for Astrophysics, 60 Garden Street, Cambridge, Massachusetts 02138
}

(Received 24 June 1992)

\begin{abstract}
A simple and accurate procedure for calculating the rate of double photoionization of "two-electron" systems at very high photon energies is presented. Arguments are given to support the validity of the method used. In particular, we show that the many-body perturbation theory diagrams depend asymptotically on the choice of the gauge for the electric dipole operator. The ratio of double-to-single ionization is calculated to be $1.68 \%$ in agreement with the recent synchrotron measurements. For $\mathrm{H}^{-}$ and $\mathrm{Li}^{+}$, we predict ratios of $1.51 \%$ and $0.89 \%$, respectively.
\end{abstract}

PACS number(s): $32.80 . \mathrm{Fb}, 32.70 . \mathrm{Cs}, 32.30 . \mathrm{Rj}$

There has been increased activity in the past year on double ionization of atoms by light absorption. Levin et al. [1] measured the rate for double ionization of $\mathrm{He}$ atom by synchrotron light at an energy of $2.8 \mathrm{keV}$. They found it to be $1.6 \% \pm 0.3 \%$ of the single ionization cross section in agreement with recent calculations using many-body perturbation theory (MBPT) by Ishihara, Hino, and McGuire (IHM) [2] which yielded a ratio of $1.6 \%$. IHM argued that the success of earlier calculations [3-6] in predicting a similar ratio for double-to-single ionization was fortuitous as they all omitted a significant part of the physics of the process.

This omitted but seemingly substantial contribution to the total ionization ratio arises, in the language of MBPT, from the strong residual electron-electron interaction following the absorption of photons by one electron in the ground state. This process, known as the two-step one (TS1), was calculated by IHM to produce a significant ionization amplitude as a function of photon energy.

In this Rapid Communication, we argue for the general validity and accuracy of the previous results and show that in the limit of high photon energies, the electronelectron correlation in the initial-state wave function is all that is needed to account for the observation, provided the acceleration form of the dipole operator is used.

It was shown by Kabir and Salpeter [7] that the asymptotic limit of the single ionization oscillator strength of helium, leaving behind the ground ionic state of $\mathrm{He}^{+}(1 s)$, behaves as

$$
\frac{d f^{+}}{d \varepsilon} \sim C(1 s)(2 \varepsilon)^{-7 / 2}\left(1-\frac{2 \pi}{(2 \varepsilon)^{1 / 2}}\right)
$$

where $\varepsilon$ is the energy available to the photoelectron in atomic units and $C(1 s)$ is a proportionality constant whose value was determined to be 287.6. Dalgarno and Stewart [5] showed that using the acceleration form of the electric dipole operator, a simple formula is obtained for this constant in the high-energy photon limit as

$$
C(n s)=\frac{512 \pi Z^{2}}{3}\left|\left\langle\Psi\left(r_{1}, r_{2}\right)\left|\delta\left(r_{2}\right)\right| u_{n s}\left(r_{1}\right)\right\rangle\right|^{2},
$$

where $Z$ is the nuclear charge, $u_{n s}(r)$ is the usual hydrogenic radial wave function, and $\Psi\left(r_{1}, r_{2}\right)$ is the initial ground wave function for the two-electron atom or ion. They obtained an asymptotic dependence of $d f^{+} / d \varepsilon$ $\sim C(1 s)(2 \varepsilon)^{-7 / 2}$ upon using the Born wave function for the final state. (The extra weak dependence on energy [see Eq. (1)] comes from the logarithmic phase of the Coulomb function.) It is important to note that using the length form of the electric dipole operator with the Born wave function yields an incorrect $\varepsilon^{-5 / 2}$ dependence of the oscillator strength with energy. In addition, for the interesting cases of $\mathrm{He}$ and $\mathrm{H}^{-}$, the dipole mixing of the excited states (caused by the angular momentum degeneracy) may be neglected as the higher angular momentum states contribute an additional factor of $\varepsilon^{-l}$ to Eq. (1). Using a sum rule, Dalgarno and Stewart showed that

$$
\begin{aligned}
C & \equiv \sum_{n} C(n s)+\int_{0}^{\infty} C\left(\varepsilon^{\prime} s\right) d \varepsilon^{\prime} \\
& =\frac{512 \pi Z^{2}}{3}\left\langle\left|\Psi\left(r_{1}, 0\right)\right|^{2}\right\rangle .
\end{aligned}
$$

Equation (3) is particularly useful in that (a) only a "good" representation of the initial-state wave function is needed to calculate accurately the sum and (b) this sum includes the contribution from double ionization.

The ratio of the double-to-single ionization cross section is given asymptotically by

$$
R=1-\frac{\sum_{n} C(n s)}{C}
$$

We have calculated $C(n s)$ and $C$ using for $\Psi\left(r_{1}, r_{2}\right)$ the parametric correlated representation of Hart and Herzberg [8] for the ground state of $\mathrm{H}^{-}, \mathrm{He}$, and $\mathrm{Li}^{+}$. The results are listed in Table I. We find $C=309.15,6.98$, and 2484.56 and $C(1 s)=287.30,4.12$, and 2557.35 , respectively, for $\mathrm{He}, \mathrm{H}^{-}$, and $\mathrm{Li}^{+}$. The higher $C(n s)$ fall off as $n^{-3}$. Table I shows a large fractional oscillator strength in the $\mathrm{H}(n=2)$ state. It is caused in part by the presence of the broad shape resonance just above the $\mathrm{H}(n=2)$ threshold which decays predominantly to the $\mathrm{H}(n=2)$ continuum [9]. Also shown in Table $\mathbf{I}$ are the results of the independent-particle approximation for the ground-state wave function of $\mathrm{He}$ using a screened charge of $\left(Z-\frac{5}{16}\right)$. The result $R=0.73 \%$ is the shake limit for which IHM give a value of $R=0.55 \%$. 
TABLE I. Ratio of $C(n s) / C$ and $R$ for negative hydrogen ion, helium, and lithium singly charged positive ion.

\begin{tabular}{rllcc}
\hline \multicolumn{1}{c}{$n$} & \multicolumn{1}{c}{$\mathrm{H}^{-}$} & \multicolumn{1}{c}{$\mathrm{He}$} & $\mathrm{He}$ (shake) & $\mathrm{Li}^{+}$ \\
\hline 1 & 0.5910 & 0.9294 & 0.9786 & 0.9715 \\
2 & 0.3885 & 0.0445 & 0.0110 & 0.0156 \\
3 & 0.0031 & 0.0055 & 0.0018 & 0.0024 \\
4 & 0.0011 & 0.0018 & 0.0006 & 0.0008 \\
5 & 0.0005 & 0.0008 & 0.0003 & 0.0004 \\
6 & 0.0003 & 0.0005 & 0.0002 & 0.0002 \\
$\geq 7$ & 0.0002 & 0.0007 & 0.0002 & 0.0002 \\
$R(\%)$ & 1.51 & 1.68 & 0.730 & 0.890 \\
\hline \hline
\end{tabular}

The double-to-single ionization ratios $R$ are respectively $1.68 \%, 1.51 \%$, and $0.89 \%$ for $\mathrm{He}, \mathrm{H}^{-}$, and $\mathrm{Li}^{+}$. The value for helium agrees with the experimental observation and the MBPT calculations. (The value of $R$ for helium differs from that obtained from the numerical data of Dalgarno and Ewart [6] which appear to contain an error for He.)

In the following, we present arguments to demonstrate that the leading term in the expansion of inverse powers of energy of the electric dipole matrix element in the length gauge will be either exactly zero or give the wrong asymptotic dependence with photon frequency. To this end, we assume that the final one-electron radial wave function can be cast in the form of an energy normalized spherical Bessel function of unit angular momentum,

$$
\psi_{\varepsilon}=\left(\frac{2 k}{\pi}\right)^{1 / 2} r j_{1}(k r),
$$

where $\varepsilon=k^{2} / 2$ in atomic units. Since we are interested in the limit of high frequency (or momentum), matrix elements involving the dipole operator connecting the final state to the ground state $\psi_{i}, M=\left\langle\psi_{\varepsilon}|d| \psi_{i}\right\rangle$ will oscillate rapidly and the important contributions will therefore come from $k r=1$. It therefore seems plausible to expand the initial bound wave function $\psi_{i}$ near the nucleus [10]

$$
\psi_{i}=a_{0} r+a_{1} r^{2}+\cdots \text {. }
$$

Then, in the length gauge, $d=r$, and

$$
M^{L} \propto k^{1 / 2} \int_{0}^{\infty} j_{1}(k r) r^{3}\left(a_{0}+a_{1} r+\cdots\right) d r .
$$

Making the substitution $\rho=k r$,

$M^{L} \propto k^{-7 / 2}\left(a_{0} \int_{0}^{\infty} \rho^{3} j_{1}(\rho) d \rho+a_{1} k^{-1} \int_{0}^{\infty} \rho^{4} j_{1}(\rho) d \rho\right)$.

Note that the first term of the expansion in (7b) involves an integral which is identically zero [11]. We therefore have to go to the next term to obtain the leading energy exponent,

$$
M^{L} \simeq k^{-9 / 2} a_{1} \int_{0}^{\infty} \rho^{4} j_{1}(\rho) d \rho \propto k^{-9 / 2} .
$$

The photoionization cross section now behaves asymptotically as

$$
\sigma \propto \varepsilon\left|M^{L}\right|^{2} \sim \varepsilon^{-7 / 2},
$$

a result known for the photoionization of hydrogen [12]. In the acceleration gauge, $d-1 / r^{2}$ and

$$
M^{A} \propto k^{-1 / 2}\left(a_{0} \int_{0}^{\infty} j_{1}(\rho) d \rho+a_{1} k^{-1} \int_{0}^{\infty} \rho j_{1}(\rho) d \rho\right) .
$$

It is straightforward to see that the leading term in $M^{A}$ already contains the correct nonvanishing asymptotic behavior as the integral over $j_{1}(\rho)$ reduces to a ratio of $\Gamma$ functions [11] and therefore

$$
\sigma \propto \varepsilon^{-3}\left|M^{A}\right|^{2}-\varepsilon^{-7 / 2}
$$

An alternative argument can be given to demonstrate that the derivation of Eqs. (2)-(4) is asymptotically exact. If we expand the approximate wave function of helium in the final state, $\psi_{\varepsilon}^{0}\left(\mathbf{r}_{1}, \mathbf{r}_{2}\right)$, in terms of the complete set of exact eigenfunctions $\psi_{n}\left(r_{1}, r_{2}\right)$

$$
\psi_{\varepsilon}^{0}=\psi_{\varepsilon}+\sum_{n} a_{n} \psi_{n}
$$

where $\psi_{\varepsilon}$ is the exact eigenfunction of the two-electron Hamiltonian in the final state, we may write the transition matrix element $Q_{0 \varepsilon}$ as

$$
\begin{aligned}
& Q_{0 \varepsilon}^{L}=Q_{0 \varepsilon}+\sum_{n} a_{n} Q_{n \varepsilon}, \\
& Q_{0 \varepsilon}^{V}=Q_{0 \varepsilon}+\sum_{n} a_{n} Q_{n \varepsilon} \frac{E_{n}-E_{0}}{\varepsilon+I-E_{0}}, \\
& Q_{0 \varepsilon}^{A}=Q_{0 \varepsilon}+\sum_{n} a_{n} Q_{n \varepsilon} \frac{\left(E_{n}-E_{0}\right)^{2}}{\left(\varepsilon+I-E_{0}\right)^{2}},
\end{aligned}
$$

where $I$ stands for the ionization potential and the superscripts $L, \bar{V}$, and $A$ refer to the length, velocity, and acceleration forms of the electric dipole operator [13]. We assume that $a_{n}$ are small such that higher-order corrections are negligible. Note also that the matrix element $Q_{0 \varepsilon}$ is defined such that it is independent of the choice of the gauge for the transition operator, i.e., it is defined as the matrix element of $d$ between the "exact" initial discrete function $\Psi$ and the continuum wave function $\psi_{\varepsilon}$.

In the limit as $\varepsilon \rightarrow \infty$,

$$
\begin{aligned}
& Q_{\delta_{\varepsilon}}^{L}=Q_{0 \varepsilon}+\sum_{n} a_{n} Q_{n \varepsilon}, \\
& Q_{0 \varepsilon}^{V}=Q_{0 \varepsilon}+\frac{1}{\varepsilon} \sum_{n} a_{n} Q_{n \varepsilon}\left(E_{n}-E_{0}\right), \\
& Q_{0 \varepsilon}^{A}=Q_{0 \varepsilon}+\frac{1}{\varepsilon^{2}} \sum_{n} a_{n} Q_{n \varepsilon}\left(E_{n}-E_{0}\right)^{2} .
\end{aligned}
$$

The above relations assert that for large photon energies the error in the length form of the matrix element remains unaltered whereas in the velocity and acceleration forms the error drops as $\varepsilon^{-1}$ and $\varepsilon^{-2}$, respectively.

IHM claimed that this asymptotic method ignores the contribution from a specific group of MBPT diagrams. We now illustrate how the different MBPT diagrams are gauge dependent, i.e., their behavior with energy (at least asymptotically) depends on the different forms of the dipole operator.

The definition of the TSI amplitude as 


$$
A_{\mathrm{TS} 1}=\left\langle\psi_{s}\left|\frac{1}{r_{12}} G_{s}{ }^{+} d\right| \Psi\right\rangle,
$$

allows us to extract the asymptotic energy dependence of the TS1 process, using for the final state continuum wave function

$$
\psi_{\varepsilon} \sim k^{-1 / 2}\left(k r_{1}\right) j_{1}\left(k r_{1}\right) f\left(k^{\prime} r_{2}\right)+(1 \rightarrow 2)
$$

where $d=1 / r_{1}^{2}$. We write the out-going wave radial Green function as

$$
\begin{aligned}
G_{\varepsilon}^{+}\left(r_{1}, r_{1}^{\prime}\right) & =k r_{1} j_{1}\left(k r_{1}\right) e^{i \mathbf{k} \cdot r_{1}} \\
& \equiv F\left(k r_{1}, k r_{1}^{\prime}\right),
\end{aligned}
$$

where the notations have the usual meaning. For the electron-electron interaction potential, we use the monopole approximation following Vegh and Burgdorfer [14].

By making the substitution of the conjugate product $\rho=k r_{1}$ and $\rho^{\prime}=k r_{1}^{\prime}$, the Green function introduces no energy factor to the asymptotic form of Eq. (15). The electron-electron interaction and the electric dipole operators contribute a combined $k^{3}$ dependence upon the change of variable. Integrals over $r_{1}$ and $r_{1}^{\prime}$ give a $k^{-2}$ energy factor. The other integral over $r_{2}$ contains a piece of the final state $f\left(k^{\prime} r_{2}\right)$ and a part of the ground-state wave function. Now since the two electrons occupy very different regions of the phase space, i.e., one electron carries off essentially all the photon energy such that $k \gg k^{\prime}$, then one can make the same small-r expansion for $f\left(k^{\prime} r_{2}\right)$ as was done in Eq. (6). The initial bound wave function can also be expanded near the origin as $\Psi\left(r_{1}, r_{2}\right)$ $\simeq\left(a_{0} k^{-1} \rho\right)\left(b_{0} r_{2}\right)$ so that the $r_{2}$ integral contributes $k^{\prime} k^{-3}$ to the asymptotic form of Eq. (15).

The TS1 amplitude squared is then proportional to

$$
\left|A_{\mathrm{TS} I}\right|^{2} \propto k^{-7} k^{\prime 2} \text {. }
$$

At each photon energy, $\omega$, the total double ionization rate is proportional to the integral of the matrix element in (17) over $\varepsilon$ and $\varepsilon^{\prime}$ such that the energy conservation law $\omega+I=\varepsilon+\varepsilon^{\prime}$ is satisfied. (Recall that $k^{2}=2 \varepsilon$ and $k^{\prime 2}$ $=2 \varepsilon^{\prime}$.) Owing to this fact, one integral goes away and the remaining integral over $\varepsilon$ can be carried out analytically to give the "partial" TS1 cross section as

$$
\sigma_{\mathrm{TS} 1}^{A} \propto \omega^{-3} \int_{\omega} \varepsilon^{-7 / 2} \varepsilon d \varepsilon \sim \omega^{-3} \omega^{-3 / 2} \approx \varepsilon^{-9 / 2},
$$

whereas the same analysis using the length form of the dipole operator yields $\sigma f_{s !}-\varepsilon^{-7 / 2}$. [This point was independently suggested by Amusia (see Ref. 16 of IHM).] Thus, in the acceleration gauge, the TS1 diagram does not contribute to the asymptotic form of the single and double ionization cross sections.

It is worthwhile to note that in the velocity gauge $[4,7]$, the accuracy of the results rests crucially on the cusp condition at the nucleus, i.e., $\left(\partial \Psi / \partial r_{2}\right)_{r_{2}}=0$. For helium, this condition for the cusp is equal to $\left(\partial \Psi / \partial r_{2}\right)_{r_{2}}=0$ $=-2 \Psi\left(r_{1}, r_{2}=0\right)$. Thus if the ground-state correlated wave function deviates from the cusp ratio of -2 , the results can vary substantially [4].

In summary, we have shown, using simple arguments, that asymptotically exact estimates for the double photoionization rates of two-electron systems can be obtained. This method recognizes that the region for the highenergy photon interaction with the electrons is confined to a small volume near the nucleus of the order $r \sim \varepsilon^{-1 / 2}$ so that the only relevant dynamic two-electron correlation is contained in the initial ground-state configuration. It is demonstrated that the MBPT diagrams are asymptotically gauge dependent and that the TS1 "partial" cross section diminishes more rapidly with photon frequency in the acceleration gauge than in the length gauge.

Note added. After this work was completed, we became aware of a new experiment [15] in which the photon energy range was extended considerably beyond $2.8 \mathrm{keV}$ ( 2 to $12 \mathrm{keV}$ ) with the result that to within experimental error the ratio is a constant $1.5 \pm 0.2 \%$.

This work was supported by the U.S. Department of Energy, Division of Chemical Sciences, Office of Basic Energy Sciences, Office of Energy Research. Conversations with Joachim Burgdorfer, and correspondence with and comments by Jim McGuire on an earlier version of the manuscript are gratefully acknowledged.
[1] J. C. Levin, D. W. Lindle, N. Keller, R. D. Miller, Y. Azuma, N. Berry Mansour, H. G. Berry, and I. A. Sellin, Phys. Rev. Lett. 67, 968 (1991).

[2] T. Ishihara, K. Hino, and J. H. McGuire, Phys. Rev. A 44, R6980 (1991).

[3] F. W. Byron and C. J. Joachain, Phys. Rev. 164, 1 (1967).

[4] T. Aberg, Phys. Rev. A 2, 1726 (1970).

[5] A. Dalgarno and A. L. Stewart, Proc. Phys. Soc. London 76, 49 (1960).

[6] A. Dalgarno and R. W. Ewart, Proc. Phys. Soc. London 80, 616 (1962).

[7] P. K. Kabir and E. E. Salpeter, Phys. Rev. 108, 1256 (1957).

[8] J. F. Hart and G. Herzberg, Phys. Rev. 106, 79 (1957).
[9] H. R. Sadeghpour, C. H. Greene, and M. Cavagnero, Phys. Rev. A 45, 1587 (1992).

[10] A. R. P. Rau and U. Fano, Phys. Rev. 162, 68 (1967).

[11] G. N. Watson, A Treatise on the Theory of Bessel Functions, 2nd ed. (Cambridge Univ. Press, Cambridge, 1944), Sec. 13.2.

[12] H. A. Bethe and E. E. Salpeter, Quantum Mechanics of One- and Two-Electron Atoms (Plenum/Rosetta Edition, New York, 1977), Sec. 71.

[13] A. Dalgarno and J. T. Lewis, Proc. Phys. Soc. London 69, 285 (1956).

[14] L. Vegh and J. Burgdorfer, Phys. Rev. A 42, 655 (1990).

[15] I. A. Sellin (private communication). 